\title{
The Study of DC Conductivity for Polyaniline-polymannuronate Nano Composites
}

\author{
C. Basavaraja, Y. Veeranagouda, ${ }^{\dagger}$ Kyoung Lee. ${ }^{\dagger}$ R. Pierson. M. Revanasiddappa, ${ }^{\ddagger}$ and Do Sung Huh ${ }^{\star}$ \\ Department of Chemistry and Institute of Functional Material, Inje University, Kimhae, Kyungnam 621-749, Korea \\ ${ }^{*} E$-mail: chemhds@inje.ac.kr \\ "Department of Microbiology, Changwon National University, Changwon, Kyungnam 641-773, Korea \\ 'Department of Chemistry, PES School of Engineering, Bangalore 560-100, India \\ Received Angust 12, 2008
}

\begin{abstract}
Conducting polyaniline-polymannuronate (P $\Lambda$ NI-PM) composites have been prepared by in situ deposition technique by placing different wt. $\%$ of fine grade powder of polymannuronate $(5,10,15$, and $25 \%)$ during polymerization of polyaniline. The oxidative polymerization of aniline was carried by using ammonium persulfate as oxidant. Further the temperature-dependent DC conductivity of PANI-PM composites was studied within the range of $300 \leq \mathrm{T} \leq 500 \mathrm{~K}$. The conductivity data shows an evidence for the transport properties of PANI-PM composites. This study in tum supports the strong interaction of PANI with PM in the composites. Based on the DC conducting properties the composites signify the future development of new materials for the creation of new bio-nanocomposites that has a multi-component and multifunctional material.
\end{abstract}

Key Words : Polyaniline, Polymannuronate, Nano-composite, Nanoscopic textures

\section{Introduction}

Intrinsically conducting polymers (ICP), ${ }^{12}$ namely polyaniline, polypyrrole, etc., are also known as "synthetic metals" which are of extensive theoretical and experimental studies in recent years. ${ }^{3}$ Since the discovery of their metallic conductivity by addition of suitable dopants ${ }^{+}$provide them promising materials for microelectronic devices such as light-emitting diodes, ${ }^{5.6}$ thin-film transistors, ${ }^{7}$ gas sensors, ${ }^{8}$ and organic transparent electrodes. ${ }^{9.10}$ The dispersion of ICP, especially PANI with thermoplastic polymers to form blends has proven to be one of the useful methods and have found several technical applications in metal corrosion protection and printed-board circuit." ${ }^{11.12}$ Various efforts have been directed toward enhancing PANI's electroactivity ${ }^{13-15}$ either by introducing acidic groups into the PANI chains ${ }^{15.16}$ or doping PANI with negatively charged polyelectrolytes. ${ }^{17}$

Recent advancements in the development of fabrication technology, which has brought research endeavors to the micro or nano scale in order to obtain inexpensive signal processing systems, have led to the invention of a variety of novel biomedical sensors. ${ }^{12,13}$ Polysaccharides are the key elements for a number of biomaterials and biopolymers. The introduction of biopolymers in the PANI matrix is very easy because the biopolymers either partially or completely soluble in aqueous solvents. ${ }^{18}$ Polymannuronate (PM) is a good biopolymer for the purpose since it has a well-defined chemical composition; it has therefore attracted special interest in biomedical research. PM is a component of alginate and has been shown to possess many medical and phannaceutical applications. ${ }^{19-21}$ In vivo animal models have now revealed the immunologic potential of PM in such diverse areas as the protection against lethal bacterial infections and irradiation, and increasing non-specific immunity. ${ }^{22}$
$\mathrm{PM}$ is present in the form of a block co-polymer in alginate, which is a naturally occurring acidic linear polysaccharide. The molecular chain of polymannuronic acid units of PM forms a flat, ribbon-like molecule stabilized by the formation of an intramolecular hydrogen bond. Recently, Liew et $a l^{23}$ has shown that the matrix tablets prepared from high viscosity alginate (rich in mannuronic acid) enhanced the drug release rate in acidic phase. Since PM has free hydroxyl groups and negatively charged carbonyl groups, an attempt was made here to synthesize conducting composites by doping PM with PANI. In this report, polymer composites containing PANI by placing different wt. $\%$ of fine grade powder of PM $(5,10,15$, and $25 \%)$ were synthesized by chemical polymerization of aniline in an aqueous solution using of ammonium persulfate as oxidant. The enhancement of the electrical conductivity in the temperature range $300-500 \mathrm{~K}$ shows a semiconducting behavior with a negative temperature coefficient of resistivity (TCR). Further the apparent activation energy was determined Arrhenius equation show the dependence of the thermal rate process of electron transport within these temperature ranges. The increase in the activation energy may be attributed to the increase in the interchain distance with the increase in the PM concentration.

\section{Experimental}

All chemicals used for the synthesis of PANI and its composites were purchased from Sigma-Aldrich and used without further purification. PM was obtained from bacteria whose detailed procedure has been described previously. ${ }^{24}$ All solutions were prepared using deionized water. A weighed amount of PM powder was dissolved in $200 \mathrm{~mL}$ distilled water, after which $2.0 \mathrm{~mL}$ of aniline was injected into this suspension; this suspension was stirred magnetically for $2 \mathrm{~h}$. 


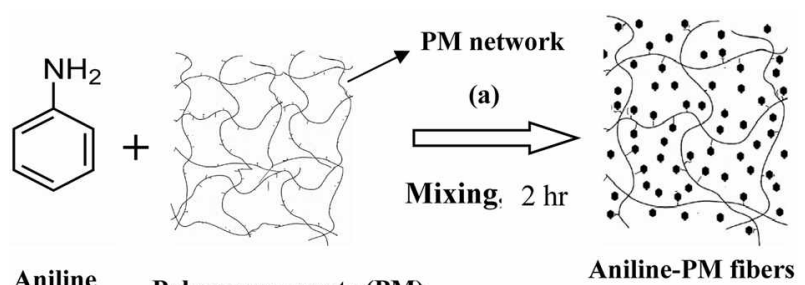

Aniline Polymannuronate (PM)

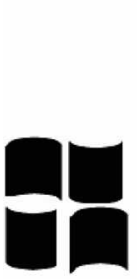

PANI-PM

Thin films

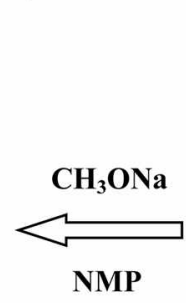

(c)

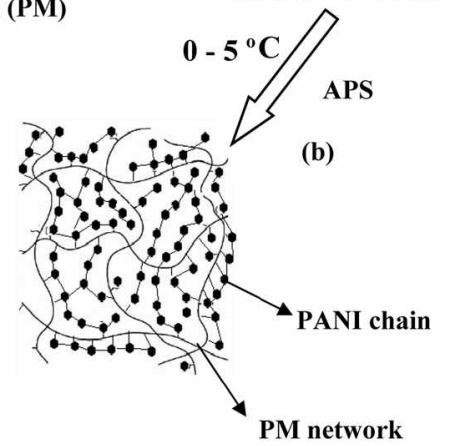

Figure 1. Polymerization of $\mathrm{PAN}$ in the presence of PM microgels to form PANI-PM compositc. (a) Incorporation of Aniline into the PM network. (b) Polymerization of aniline-P'M to form PANI-P'M composite. (c) Formation of PANI-PM thin films

Afterward, 0.1 M of APS was slowly added to the mixture with constant stirring, and the reaction was performed for about $5 \mathrm{~h}$ at $0{ }^{\circ} \mathrm{C}$. The precipitated composite was filtered and rinsed with $1 \mathrm{M} \mathrm{HCl}$ and distilled water. The target mass loading of $\mathrm{PM}$ in the composites varied from 5 to $25 \mathrm{wt} \%$. PANI homopolymer was synthesized under the same conditions for comparison with the composites. The composites are abbreviated as PANI-y, where $y$ refers to the wt $\%$ of PM powder used in the polymerization reaction.

Figure 1 depicts a schematic model for the formation of the PANI-PM composite structure. After mixing aniline with the solution containing PM, the aniline-PM complex, a slightly brown-colored solution, began to form during mixing. Following the addition of APS to this reaction mixture, the PANI-PM composite structure was formed. The figure illustrates how the microgels structure of PM stabilizes the dispersion of PANI. The fibrillar structure of PM adsorbs the aniline to form the aniline-PM complex after aniline polymerization in order to obtain the PANI-PM composite, withholding the chain of PANI into the PM matrix. Thus, a modified uniform surface of PANI-PM was formed. The resulting precipitate was filtered and washed thoroughly with dejonized water and acetone. It was then dried under vacuum for $24 \mathrm{~h}$ in order to achieve a constant weight. The characterization of the powder samples and the preparation of thin films of the obtained composites were described in our earlier research. ${ }^{2526}$ The DC electric measurements of the obtained composite films were performed within the temperature range of $300-500 \mathrm{~K}$ using the four-probe technique with a Keithly 224 constant current source and a Kejthly 617 digital electrometer.

\section{Results and Discussion}

DC electrical properties of PANI-PM composites. To

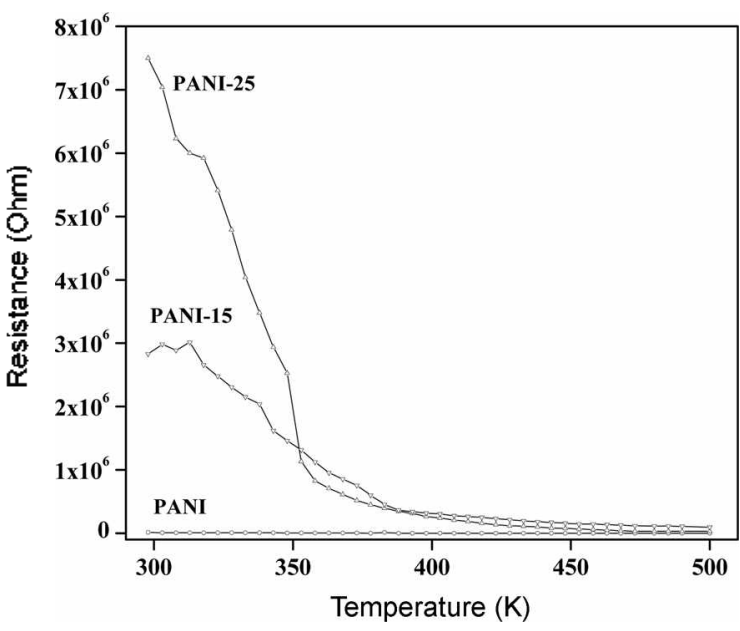

Figure 2. Temperature dependence of electrical resistance for PANI, PANI-15 and PANI-25.

investigate the charge transport mechanism in the PANI-PM composites, electrical conductivity's temperature dependence is studied for all compositions. Figure 2 shows the variation of electrical resistance with temperature for PANI, PANI-15 and PANI-25. The electrical resistance decreased exponentially with temperature which is similar to semiconducting behavior and it rises with the increase in the content of PM. The temperature coefficient of resistivity (TCR) was determined from the variation of electrical resistivity with temperature using the following relation: ${ }^{32}$

$$
\operatorname{TCR}=\left(\frac{1}{\rho\left(T_{1}\right)}\right)\left(\frac{\Delta \rho}{\Delta T}\right)
$$

where $\Delta \rho=\rho\left(T_{1}\right)-\rho\left(T_{2}\right)$, and $\Delta T=T_{2}-T_{1}$. Table 1 shows the calculated TCR values for different composites. They have negative values and decreases with temperature as the content of PM increases. The increase of conductivity with increasing temperature indicates the composites' semiconducting behavior. The results of conductivity in the composites suggest that PM wields a positive influence on the composites' conducting property, which is triggered by the mobility of PM counterions at a higher temperature. The molecular dispersion of PM in the solution results in a micro-gel state, which molds the particles in a continuous electron path generating an increased number of active sites for the charge transfer through the interface inside the electrode; this is achieved through constant contact with the PANI matrix. The PM's molecular chain is flat and ribbon-

Table 1. Calculated values of the temperature coefficient of resistivity (TCR) for $P A N I, P \wedge N I-15$, and PANI-25

\begin{tabular}{clll}
\hline \multirow{2}{*}{$\begin{array}{c}\text { Temperature } \\
(\mathrm{K})\end{array}$} & \multicolumn{3}{c}{ Temperature coefficient of resistivity (TCR) } \\
\cline { 2 - 4 } & PANI & PANI-15 & PANI-25 \\
\hline $290-350$ & -0.0094 & -0.0135 & -0.0139 \\
$350-400$ & -0.017 & -0.0140 & -0.0147 \\
$400-450$ & -0.01 & -0.0165 & -0.0176 \\
$450-500$ & -0.002 & -0.0027 & -0.00297 \\
\hline
\end{tabular}




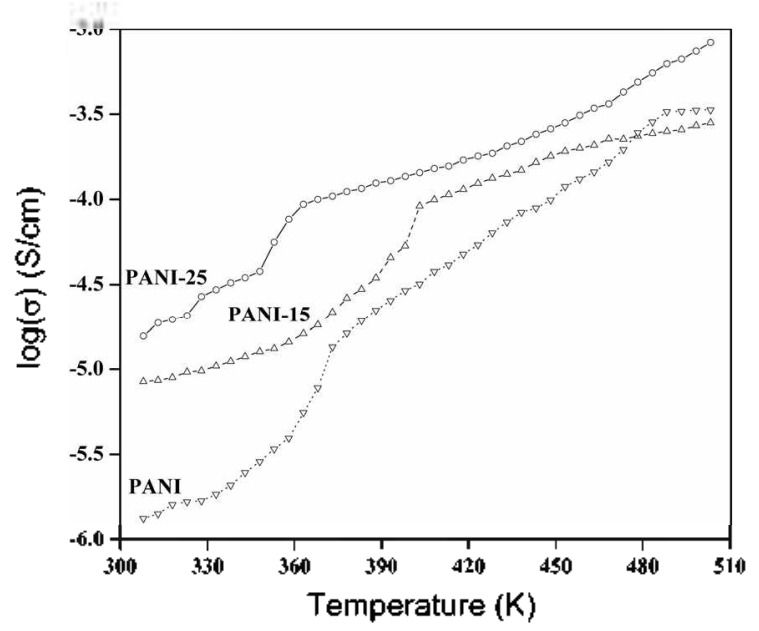

Figure 3. Temperature-dependent DC electrical conductivity of PANI, PANI-15, and PANI-25 composites.

like, and its conformation is stabilized by the formation of an intramolecular hydrogen bond in the solution. As a result, the PANI-PM composite macromolecules display very rigid and extended pattems. These form a continuous electron path by charge transfer at the interface between the electrodes. ${ }^{25-30}$ The formation of a chain structure containing PANI particles aligned in the form of interspersed chains with regions ${ }^{11.31-41}$ results in the increased conductivity properties of the crystalline-like polymer.

Figure 3 shows a relation for the $\mathrm{DC}$ conductivity vs. temperature of composites with different wt $\%$ of PM. These values vary exponentially with temperature. The results show that PM has a positive influence on the temperature dependent conducting property of PANI, which may be caused by the mobility of counterions of PM at higher temperature. This is attributed to the modification of bulk morphology of PANI by PM. Figure 4 shows a relation for logarithm of conductivity vs. temperature inverse for PANI, PANI-15 and PANI-25 which is exactly a reverse of Figure 3.

The conductivity behavior depending on temperature can

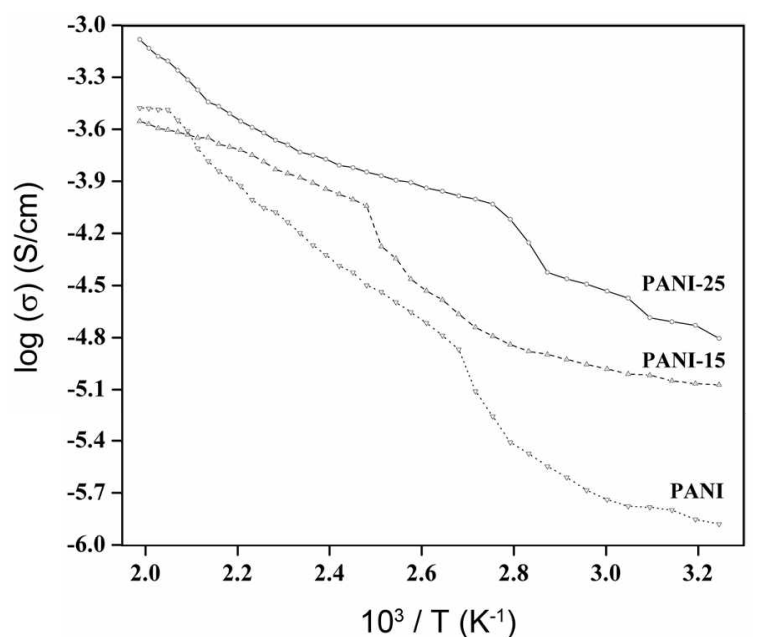

Figure 4. Inverse of temperature dependence of conductivity in logarithm for PANI, PANI-15 and PANI-25. be ascribed by two mechanisms for electrical conduction in the composites. The reason for the increase in the conductivity is attributed to the thennal energy at higher temperature to excite electrons to the conduction band (thermal process). Such behavior can be expressed by the Arrhenius equation: $:^{33}$

$$
\sigma=\sigma_{0} \exp \left(\frac{-E_{a}}{k_{\mathrm{B}} T}\right)
$$

where $\sigma$ is the $\mathrm{DC}$ conductivity, $\sigma_{0}$ is constant for a material and $k_{\mathrm{B}}$ is the Boltzmann constant, $T$ is absolute temperature, and $E_{\beth}$ is the activation energy. The other mechanism takes place in the low-temperature region ie. around $300-350 \mathrm{~K}$ and is attributed to the hopping process, in which the excited electrons in this region lose their ability transition to the conduction band with lowering temperature. Thus, the electron attempts to find a state of similar energy by tunneling beyond its nearest neighbors to hop into more distant sites. This hopping leads to a greater selection of possible energy levels of electrons. The behavior of DC conductivity in this region is called variable range hopping $\left(\sigma_{v}\right)$, which is expressed in Mott's theory as follows. ${ }^{33.34}$

$$
\sigma_{1}=\frac{\sigma_{0}}{T^{1 / 2}} \exp \left(-A T^{-1 / 4}\right)
$$

where $A$ is Arrhenius constant. The values of $E_{a}$ was determined from the slopes of the fitted lines as in Figure 4 (best fit of Arrhenius curves), and they are indicated in Table 2 at different temperature ranges. These values show the

Table 2. Calculated values of the activation energy for PANI, PANI-15, and PANI-25

\begin{tabular}{cccc}
\hline \multirow{2}{*}{$\begin{array}{c}\text { Temperature } \\
\text { (K) }\end{array}$} & \multicolumn{3}{c}{ Activation energy $(\mathrm{kcal} / \mathrm{mol}) /\left(10^{3}\right)$} \\
\cline { 2 - 4 } & PANI & PANI-15 & PANJ-25 \\
\hline $300-400$ & $(2.54 \pm 0.3)$ & $(5.56 \pm 0.25)$ & $(7.26 \pm 0.25)$ \\
$400-450$ & $(7.96 \pm 0.25)$ & $(7.36 \pm 0.2)$ & $(10.65 \pm 0.32)$ \\
$450-500$ & $(10.67 \pm 0.26)$ & $(12.85 \pm 0.15)$ & $(14.87 \pm 0.17)$ \\
\hline
\end{tabular}

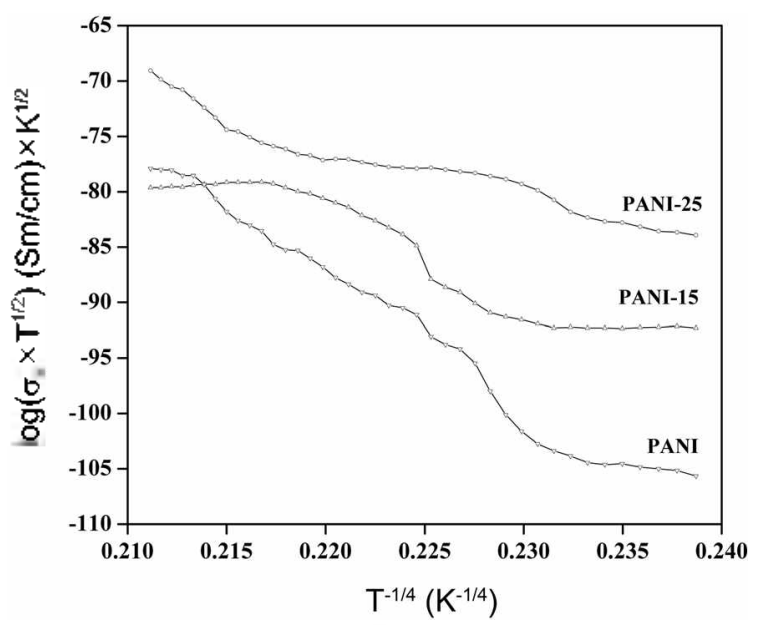

Figure 5. Plot of $\log \left(\sigma \mathrm{T}^{1 / 2}\right)$ as a function of $\mathrm{T}^{-14}$ for $\mathrm{PANI}, \mathrm{PANI}-15$ and PANT-25. 
dependence of the thermal rate process of electron transport within these temperature ranges. The difference in the values of the apparent activation energy (Table 2) for the three temperature ranges show different electrical mechanisms that are taking place in the bulk of these composites.

Figure 5 presents the relation of $\log \left(\sigma_{v} T^{12}\right)$ versus $T^{1 / 4}$ for three different composites. This figure shows a nearly linear relation within the temperature range which supports the assumed mechanism. This observation is consistent with Mott's variable range hopping process. ${ }^{32-34}$ Overall the dependence on temperature of the dc electrical conductivity of the composites is similar to semiconducting behavior with negative TCR. The electrical conductivity of these composites increases with temperature in all these composites as the PM concentration is increased, and this exhibits a thermal electron process (high-temperature region) and a hopping process (low-temperature region). The apparent activation energy shows a pronounced effect with the increase in the content of PM with temperature. By our study of this report, the synthesized composites are thermally more conducting than PANI and have a stable DC conductivity which changes with temperature.

\section{Conclusion}

PANI-PM composites were synthesized by the chemical method using aniline in an aqueous media containing welldissolved PM. The DC conductivity was studied within the temperature range of $300-500 \mathrm{~K}$. The increase in conductivity suggests that the PM acts as a suitable material for making PANI composites. Understanding the utilization of PM as a functional material may ultimately allow polysaccharides with designed architecture to be utilized in a host of biomimetic soft nanotechnology applications including sensors and advanced materials. The method described here may be useful for developing new applications of these nano-composite films in molecular electronics and other fields. The potentials offered by the PANI-PM host matrix may be exploited in the development of biosensors based on microorganisms.

Acknowledgments. This work was supported by the Korea Research Foundation Grant funded by the Korean Government (MOEHRD) (KRF-00042007070-00).

\section{References}

1. Skotheim, A. T.; Elsenbaumer, R. L.; Reynolds, J. R. Handbook of Conducting Polymers, 2nd ed.; Marcel Dekker: New York, 1998.

2. Macdiarmid, A. G. Symth. Met, 2002, 1,125 .

3. Nalwa, H. S. Handbook of Advanced Electronic and Photonic Material Devices; Academic Press: London, 2000; Vol. 8.

4. Cao, Y; Treacy, G. M.; Smith, P.; Heeger, A. J. Appl. Phys. Lett. $1992,60,2711$

5. Miller, J. S. Ad. Mater 1993, 5, 587.

6. Novak, P.; Muller, K.; Santhanam, K. S. V.; Hass, O. Chem. Rev.
$1997,97,207$.

7. Garnier, F.; Horowitz, G.; Ping, X.; Fichou, D. Act. Mater. 1990 , 2, 592 .

8. Li, H. S.; Josowicz, M.; Baer, D. R.; Engelhard, M. H.; Janata, J. J. Electrochem. Soc. 1995, 124, 798.

9. Wang, H. L.; MacDiarmid, A. G.; Wang, Y. Z.; Gebler, D. D.; Epstein, A. J. Synth. Met. 1996, 78, 33.

10. Rose, T. L.; Antonio, S. D.; Jillson, M. H.; Kron, A. B.; Suresh, R.; Wang, F. Symh. Met. 1997, 85, 1439.

11. Cao, Y.; Colaneri, N.; Heeger, A. J.; Smith, P. Appl. Phys. Lett. $1994,65,200 \mathrm{l}$.

12. Oesterholm, J. E.; Klavetter, F.; Smith, P. Polymer 1994, 35, $13 \mathrm{I}$.

13. Shilpa, J.; Sanjay, C.; Samui, A. B.; Krishnamurhy, V. N.; Bhoraskar, S. V. Achuators B Chenical 2003, 96(1-2), 124.

14. Basavaraia, C.; Huh, D. S.; Piesson, R.; Park, S. H.; Jeon, E. J.; Revanasiddappa, M.; Basavaraja, S. Bull. Korean Chem. Soc. 2008, (accepted for publication).

15. Basavaraja, C.; Choi, Y. M.; Park, H. T.; Huh, D. S.; Lee, J. W.; Revanasiddappa, M.; Raghavendra, S. C.; Khasim, S.; Vishnuvardhan, T. K. Bull. Korean Chem. Soc. 2007, 28(7), 1104.

16. Philip, N. B.; Evelyne, S. Phys. Chem. Chem. Phys. 2000, 2, 2599.

17. Bartlett, P. N.; Wallace, E. N. K. J. Electroanal. Chem. 2000 , $486(1), 23$.

18. Oleg, A. R.; Eugenil, K.; Andreas, F. B.; Iumar, W. J. Am. Chem. Soc. 2002, $124(22), 6487$.

19. Ronald, E. P.; Roy, D. K.; Jose, P. J. Sensors and Acttators A. Phosical 1998, 64(1), 77 .

20. Zhao, H.; Liu, H.; Chen, Y.; Xin, X.; Li, J.; Hou, Y.; Zhang. Z.; Zhang, X.; Xie, C.; Geng, M. D. J. Cancer Res, 2006, 66, 8779 .

2l. Jeong, J. J.; Kim, J. H.; Kim, C. K.; Hwang, I.; Lee, K. Microbiology 2003, $149,3265$.

22. Halaas, O.; Olsen, W. M.; Veiby, O. P.; Lovhaug, D.; Skjak-Braek, G.; Vik, R.; Espevik, T. Scand. J. Inmmol. 1997, 46, 358.

23. Jahr, T. G.; Ryan, L.; Sundan, A.; Lichenstein, H. S.; Skjak-Braek, G; Espevik, T. Infect. Immun, 1997, 65, 89.

24. Liew, C. V.; Chan, L. W.; Ching, A. L.; Heng, P. W. Int. J. Pharm. $2006,309,25$.

25. Basavaraja, C.; Veeranagouda, Y.; Lee, K.; Pierson, R.; Park, S. H.; Jeon, E. J.; Huh, D. S. J. Appl. Poly Sci. 2008 (submitted for publication).

26. Basavaraja, C.; Pierson, R,; Huh, D. S. J. App. Poly Sci. 2008 , 108,1070 .

27. Basavaraja, C.; Pierson, R,; Vishnuvardhan, T. K.; Huh, D. S. Enir. Poh: J. 2008, 44, 1556.

28. Kurt, I. D.; Skjak-Braek, G.; Olav, S. Int. J. Biol. Mocronolectles 1997. 21.47.

29. Harold, L. S. Bacteriological Reviews 1975, 39(4), 516 .

30. Atkins, E. D. T.; Mackie, W.; Parker, K. D.; Smolko, E. E. Polym. Lett, 1971,9,311.

31. Feng, X.; Liu, Y.; Lu, C.; Hou, W; Zhu, J. J. Nantotechology $2006,17,3578$.

32. Ayse, G. Y.; Gok, A. Synth. Met. 2007, 157(4-5), 235.

33. Mot, N. F.; Davis, E. A. Electronic Processes in Non-Chstalline Materials; Clarendon Press: Oxford, 1979.

34. Holland, E. R.; Pomfret, S. J.; Adams, P. N.; Monakan, A. P. J. Phs;: Condens. Matter. 1996, 8, 2991.

35. Joo, J.; Long, S. M.; Pouget, J. P.; Oh, E. J.; MacDiarmid, A. G.; Epstein, A. J. P/nss, Rev. B 1998, 57, 9567.

36. Kaiser, A. K. Rep. Prog. Phys. 2001, 64, I.

37. Hauser, J. J.; Kimerling, L. C. Phys, Rev. $B$ 1975, $1 /(10), 4043$.

38. Efros, A. L.; Shlovskii, B. I. J. Phys. C 1975, 8(4), 49.

39. Sheng, P. Philos. Mag, A 1992, 6.5(3), 357.

40. Zuppiroli, L.; Bussac, M. N.; Paschen, S.; Chauvet, O.; Forto, L. Phys. Rev. B 1994, $50(8), 5196$.

41. Nair, K.; Mitra, S. S. J. Non-Cinst. Solids 1977, 24, I. 